\title{
Severe Gastroparesis Flare: Is COVID-19 the Inciting Factor?
}

\author{
Aditya Patel ${ }^{\mathrm{a}, \mathrm{d}}$, Ruhma Alia, d, Muhammad Hussain ${ }^{\mathrm{b}}$, \\ Jihad Slim ${ }^{\mathrm{b}}$, Theodore DaCosta ${ }^{\mathrm{c}}$
}

\begin{abstract}
Severe acute respiratory syndrome coronavirus 2 (SARS-CoV-2) infection has emerged as a public health hazard. It is commonly associated with respiratory symptoms including fever, cough and sore throat. Viral infections have historically been implicated in the exacerbations of gastrointestinal (GI) disorders. Coronavirus disease 2019 (COVID-19) has been associated with GI manifestations including diarrhea and vomiting. However, exacerbation of underlying functional GI disorders has been rarely reported with SARS-CoV-2 infection. Herein we report a case of a 31-year-old female with acute gastroparesis flare secondary to COVID-19 which was refractory to pharmacological therapy.
\end{abstract}

Keywords: COVID-19; Functional gastrointestinal disorders; Diabetic gastroparesis; Metoclopramide

\section{Introduction}

Coronavirus disease 2019 (COVID-19) caused by infection with severe acute respiratory syndrome coronavirus 2 (SARSCoV-2) has resulted in unprecedented increase in mortality globally since 2019 [1]. Patients with COVID-19 primarily present with respiratory manifestations as the virus mainly targets the respiratory system [2]. The predilection of SARS-CoV-2 for the respiratory system may be in part due to underlying respiratory comorbidities. Gastrointestinal (GI) symptoms including nausea, vomiting, diarrhea and abdominal pain have also been reported in patients who are diagnosed with COVID-19 [3]. The etiology of the GI symptoms exhibited by patients with SARS-

Manuscript submitted December 11, 2021, accepted January 26, 2022

Published online February 16, 2022

aDepartment of Internal Medicine, Saint Michael's Medical Center, Newark, NJ, USA

bDepartment of Infectious Disease, Saint Michael's Medical Center, Newark, NJ, USA

'Department of Gastroenterology, Saint Michael's Medical Center, Newark, NJ, USA

${ }^{\mathrm{d}}$ Corresponding Author: Aditya Patel and Ruhma Ali, Department of Internal Medicine, Saint Michael's Medical Center, Newark, NJ 07102, USA. Email: adi3apr4@gmail.com and raoruhma@gmail.com

doi: https://doi.org/10.14740/jmc3883
CoV-2 infection is not well understood; however involvement of the GI tract has been reflected by fecal samples positive for SARS-CoV-2 in COVID-19 patients [4]. It is likely that chronic functional GI disorders may similarly predispose the patients to SARS-CoV-2 infection of the GI tract and may manifest as a flare-up of their underlying disorder. We report a case of a 31-year-old female with a history of gastroparesis who presented with intractable vomiting. Reverse transcriptase-polymerase chain reaction (RT-PCR) was positive for SARS-CoV-2 and the patient was thought to have a flare-up of her gastroparesis.

\section{Case Report}

\section{Investigations}

A 31-year-old female with a past medical history of insulin-dependent diabetes mellitus (IDDM) and diabetic gastroparesis presented to the emergency department (ED) with complaints of abdominal pain and intractable vomiting for the past $12 \mathrm{~h}$. She was diagnosed with diabetes 10 years back and takes insulin. Patient was diagnosed with gastroparesis via gastric emptying scan 3 years ago. Patient reported that her last flare-up was 2 years back and was admitted to the hospital for 3 days with improvement in her symptoms on metoclopramide. On this admission, the patient reported severe, constant, diffuse abdominal pain without any aggravating or relieving factors. She also reported having 10 episodes of non-bloody, nonbilious vomiting mainly containing food particles. She denied any fatigue, shortness of breath, cough, numbness in her hands and feet, fever, or chill. Review of systems was negative for any other complaint. The patient also reported she lived with her mother who tested positive for SARS-CoV-2 infection 6 days ago. Vital signs on admission showed temperature of 37.0 ${ }^{\circ} \mathrm{C}$, respiratory rate $22 / \mathrm{min}$, heart rate $90 / \mathrm{min}$, blood pressure $139 / 89 \mathrm{~mm} \mathrm{Hg}$, and saturating $98 \%$ on room air. Respiratory and cardiac examination was normal. Her abdomen was soft, diffusely tender, non-distended, without any rebound tenderness or guarding with normal bowel sounds. No hepatosplenomegaly or flank tenderness was noted.

\section{Diagnosis}

Initial labs did not show any electrolyte abnormality. A naso- 
Table 1. Laboratory Parameters

\begin{tabular}{lll}
\hline Laboratory parameters & Values & Reference range \\
\hline Sodium & 138 & $136-145 \mathrm{mmol} / \mathrm{L}$ \\
Potassium & 4.1 & $3.5-5.5 \mathrm{mmol} / \mathrm{L}$ \\
Blood urea nitrogen $(\mathrm{BUN})$ & 45 & $6-24 \mathrm{mg} / \mathrm{dL}$ \\
Creatinine & 2.6 & $0.5-1.0 \mathrm{mg} / \mathrm{dL}$ \\
White blood cell count (WBC) & 11.4 & $4.4-11 \times 10^{3} / \mu \mathrm{L}$ \\
Hemoglobin $(\mathrm{Hb})$ & 7.7 & $13.5-17.5 \mathrm{~g} / \mathrm{dL}$ \\
Hemoglobin A1c & 9.4 & $4.0-5.6 \%$ \\
D-dimer & 1,536 & $0.0-500 \mathrm{ng} / \mathrm{mL}$ \\
Ferritin & 230 & $11-307 \mathrm{ng} / \mathrm{mL}$ \\
C-reactive protein $(\mathrm{CRP})$ & 15.2 & $0.0-0.8 \mathrm{mg} / \mathrm{dL}$ \\
Lactate dehydrogenase $(\mathrm{LDH})$ & 304 & $122-222 \mathrm{U} / \mathrm{L}$ \\
\hline
\end{tabular}

pharyngeal swab for reverse transcriptase-polymerase chain reaction (RT-PCR) for SARS-CoV-2 was positive. SARS-CoV-2 virus antigen was also positive. Her inflammatory markers including D-dimer, ferritin, and lactate dehydrogenase (LDH) were markedly elevated. Her labs at the time of admission are shown in Table 1 . The electrocardiogram showed sinus rhythm with no significant ST-T wave abnormalities. The chest Xray showed multiple patchy areas of consolidation at the lung bases. The X-ray of the abdomen showed a normal bowel gas pattern with paucity of small bowel gas. Abdominal ultrasound was normal. Computed tomography (CT) scan without contrast was positive for mild changes associated with COVID-19 as shown in Figure 1.

\section{Treatment}

Patient was started on low residual small frequent meals. Tight glucose control was ensured. The gastroenterology service was consulted, and she was diagnosed with gastroparesis flare secondary to medication non-compliance. She was started on pantoprazole, ondansetron, and prophylactic anticoagulation. She was not given remdesivir or dexamethasone. Over the course of the hospital stay the patient's symptoms did not improve, and she continued to complain of nausea and vomiting. Ondansetron was switched to metoclopramide with only mild improvement of symptoms. She reported that her symptoms were significantly severe as compared to her previous gastroparesis flare and were not improving with pharmacological therapy. On day 3 the administration of metoclopramide was changed to standing medication and trimethobenzamide was added. She reported improvement in her symptoms on day 7 with her repeat RT-PCR for COVID-19 was also negative.

\section{Follow-up and outcomes}

She was discharged on day 11 with 2 weeks of anticoagulation and metoclopramide with follow-up in 2 weeks. On the follow-up gastroenterology visit the patient reported improve-

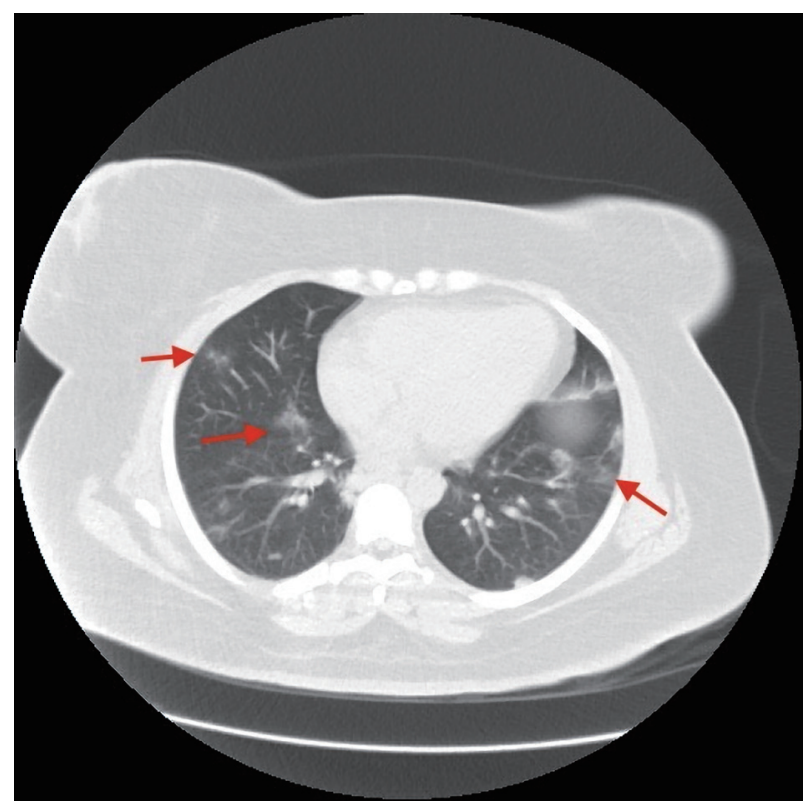

Figure 1. Mild patchy areas of consolidation bilaterally on CT scan (red arrows). CT: computed tomography.

ment in her symptoms.

\section{Discussion}

In December 2019 a new strain of coronavirus named SARSCoV-2 was discovered in China which was declared a pandemic due to the fast-spreading respiratory viral disease named COVID-19 [5]. SARS-CoV-2 belongs to the beta group of coronaviruses (CoVs) [6]. The CoVs belong to a large family of single-stranded, positive-sense ribonucleotide RNA viruses [7]. COVID-19 primarily affects the lungs and can present with self-limiting symptoms including fever, dyspnea, fatigue and dry cough. GI symptoms and renal involvement have also been reported in some patients with COVID-19 [8]. The GI symptoms include abdominal pain, nausea, vomiting and diarrhea. Patients can also harbor the virus for a period of 2 - 14 days without any symptoms [9]. Severe form of the disease can lead to acute respiratory distress syndrome, septic shock, renal failure, cardiac arrhythmias, and death making an early diagnosis substantial to prevent these complications [10]. Given that the notable percentage of COVID-19 patients present with GI symptoms, screening patients with GI manifestations for COVID-19 remains essential.

The pathogenesis of COVID-19 in the GI tract is poorly understood due to the limited knowledge of the effects of CoVs on the GI system. It is postulated that the first step of viral entry into the enterocytes occurs through the angiotensin-converting enzyme 2 (ACE-2) protein [11]. ACE-2 is mostly expressed in the vascular endothelial cells, Leydig cells and renal tubular epithelium; it is also detected in the glandular cells of gastric, duodenal, and rectal epithelium. This might be the potential point of entry of the virus into the GI tract. The virus then replicates inside the cells and is released [12]. This mechanism might also 
be the cause of inter-organ dissemination after the initial inoculation given the presence of SARS-CoV-2 receptors on multiple organ systems [13]. This can also suggest that underlying comorbidities play a role in the pathogenesis of the virus [4]. In a meta-analysis consisting of 1,576 patients, previous respiratory system pathologies were the most prevalent comorbidity reported in COVID-19 patients primarily presenting with pulmonary symptoms [14]. This might also be the reason why our patient presented solely with GI symptoms despite the evidence of pulmonary disease on imaging. SARS-CoV-2 has been detected in the stool of infected individuals upto 10 weeks after initial symptoms. Therefore COVID-19 may continue to shed the virus through stool long after the resolution of pulmonary symptoms [7]. Cytokines like interleukin 2, 10 and tumor necrosis factor $\alpha(\mathrm{TNF}-\alpha)$ have previously been involved in GI health and disease. The release of these cytokines in the GI tract of patients with COVID-19 may also be involved in the damage of the GI tract [15].

Our patient had mild pulmonary symptoms; however her underlying gastroparesis may have predisposed her to SARSCoV-2 infection of the GI tract as indicated by her symptom severity. The symptoms were refractory to pharmacological therapy and improved along the course of the COVID-19 disease. Although it is possible that her symptoms are independent of the COVID-19 infection and associated with her uncontrolled diabetes; however, the severity of the disease and no significant improvement in her symptoms after pharmacological therapy and better glycemic control, raised the question of different triggers for her exacerbation. Viruses have been implicated in pathogenesis and exacerbation of functional GI disorders. Our patient reported improvement in her symptoms after 7 days and subsequently tested negative for SARS-CoV-2. Based on her clinical course, SARS-CoV-2 is the likely scenario in this case. The proposed mechanism of viral-induced gastroparesis is through an acute injury to either interstitial cells of Cajal in the stomach or neural innervation leading to delayed gastric emptying [16]. Kim et al found that patients with ulcerative colitis (UC) and concomitant cytomegalovirus (CMV) have a higher rate of hospitalization for exacerbations as compared to UC alone [17]. Therefore, it is reasonable to suspect COVID-19 as a nidus to flare-up. Gastroparesis flare can also be caused by medications, surgery, recreational drugs, and hypothyroidism. The thyroid profile was normal in our patient. She did not report taking any new medication or recreational drugs and did not have any recent surgery. Our patient also had significant improvement in her symptoms with the natural disease course of COVID-19.

This unique case illustrates that COVID-19 can present with exacerbated symptoms of an underlying pathology and should be kept in mind in case of a flare-up of a disease. Further studies are needed to determine if patients with chronic GI pathologies are at higher risk of infection of the GI tract and to formulate a guideline-directed medical therapy.

\section{Learning points}

This case illustrates that COVID-19 can present as a severe flare-up of a chronic GI disease. Our patient had a severe gastroparesis flare-up concomitantly with a positive COVID-19 diagnosis. Improvements in her symptoms were noted with natural disease progression of COVID-19. A low threshold of suspicion for COVID-19 disease in patients with GI manifestations should be maintained especially in case of an exacerbation of an underlying chronic disorder. Further insight is required not only for early diagnosis and intervention but also to improve mortality in this patient population.

\section{Acknowledgments}

None to declare.

\section{Financial Disclosure}

None to declare.

\section{Conflict of Interest}

None to declare.

\section{Informed Consent}

The informed consent was obtained from the patient for the publication of the manuscript.

\section{Author Contributions}

Aditya Patel MD, Ruhma Ali MD and Muhammad Hussain MD: diagnosis and management of the patient, discussion, writing and drafting of the case, final approval of the case report. Jihad Slim MD and Theodore DaCosta MD: management of the patient, revision and final approval of the case report.

\section{Data Availability}

The authors declare that data supporting the findings of this study are available within the article.

\section{References}

1. Patel A, Ajayi F, Ali R, Chan KH, Slim J. Dilemma of anticoagulation therapy in mild or asymptomatic COVID-19 cases. Cureus. 2021;13(11):e19291.

2. Rothan HA, Byrareddy SN. The epidemiology and pathogenesis of coronavirus disease (COVID-19) outbreak. J Autoimmun. 2020;109:102433.

3. Huang C, Wang Y, Li X, Ren L, Zhao J, Hu Y, Zhang L, et al. Clinical features of patients infected with 2019 novel coronavirus in Wuhan, China. Lancet. 2020;395(10223):497- 
506.

4. Song J, Bhuta R, Baig K, Parkman HP, Malik Z. COVID-19 infection manifesting as a severe gastroparesis flare: A case report. Medicine (Baltimore). 2021;100(14):e25467.

5. Sohrabi C, Alsafi Z, O'Neill N, Khan M, Kerwan A, AlJabir A, Iosifidis C, et al. World Health Organization declares global emergency: A review of the 2019 novel coronavirus (COVID-19). Int J Surg. 2020;76:71-76.

6. Cheng ZJ, Shan J. 2019 Novel coronavirus: where we are and what we know. Infection. 2020;48(2):155-163.

7. Kopel J, Perisetti A, Gajendran M, Boregowda U, Goyal H. Clinical Insights into the Gastrointestinal Manifestations of COVID-19. Dig Dis Sci. 2020;65(7):1932-1939.

8. Wang D, Hu B, Hu C, Zhu F, Liu X, Zhang J, Wang B, et al. Clinical characteristics of 138 hospitalized patients with 2019 novel coronavirus-infected pneumonia in Wuhan, China. JAMA. 2020;323(11):1061-1069.

9. Wu D, Wu T, Liu Q, Yang Z. The SARS-CoV-2 outbreak: what we know. Int J Infect Dis. 2020;94:44-48.

10. Hassani AH, Beheshti A, Almasi F, Ketabi Moghaddam P, Azizi M, Shahrokh S. Unusual gastrointestinal manifestations of COVID-19: two case reports. Gastroenterol Hepatol Bed Bench. 2020;13(4):410-414.

11. Xiao F, Tang M, Zheng X, Liu Y, Li X, Shan H. Evidence for Gastrointestinal Infection of SARS-CoV-2. Gastroenterology. 2020;158(6):1831-1833.e3.
12. Glowacka I, Bertram S, Herzog P, Pfefferle S, Steffen I, Muench MO, Simmons G, et al. Differential downregulation of ACE2 by the spike proteins of severe acute respiratory syndrome coronavirus and human coronavirus NL63. J Virol. 2010;84(2):1198-1205.

13. Muus C, Luecken MD, Eraslan G, et al. Integrated analyses of single-cell atlases reveal age, gender, and smoking status associations with cell type-specific expression of mediators of SARS-CoV-2 viral entry and highlights inflammatory programs in putative target cells. bioRxiv. 2020.

14. Yang J, Zheng Y, Gou X, Pu K, Chen Z, Guo Q, Ji R, et al. Prevalence of comorbidities and its effects in patients infected with SARS-CoV-2: a systematic review and metaanalysis. Int J Infect Dis. 2020;94:91-95.

15. Holshue ML, DeBolt C, Lindquist S, Lofy KH, Wiesman J, Bruce H, Spitters C, et al. First case of 2019 novel coronavirus in the United States. N Engl J Med. 2020;382(10):929936.

16. Dignass AU, Podolsky DK. Interleukin 2 modulates intestinal epithelial cell function in vitro. Exp Cell Res. 1996;225(2):422-429.

17. Kim JJ, Simpson N, Klipfel N, Debose R, Barr N, Laine L. Cytomegalovirus infection in patients with active inflammatory bowel disease. Dig Dis Sci. 2010;55(4):10591065 . 